\title{
Estimating the Credit Quality of Chemical Companies
}

\section{Jan Henrik Wosnitza*}

Research Assistant, Institute of Business Administration, University of Muenster, Germany

In a recently published paper, Wosnitza and Leker [1] suggested a logistic regression model for estimating the rank order of international companies according to their credit quality. The aim of the article at hand is to compare the classification performances between the outcomes of the proposed model and ratings from Standard \& Poor's (S\&P's) for chemical companies. The data sample contains S\&P's ratings and financial ratios of eleven international companies from 2010. Despite small deviations, both approaches come to very similar results. Therefore, this paper validates Wosnitza's and Leker's logistic regression model and suggests to apply the equation for assessing the credit quality of chemical companies.

According to the Basel Committee on Banking Supervision [2], the logistic regression has developed into a standard method for estimating large companies' probabilities of default (PDs) from financial ratios. In order to understand how the logistic regression estimates corporate PDs, let us consider a company $k$ whose relevant default information is summarized in the vector $\vec{x}_{k}$. The logistic regression assigns a credit score between zero and one to company $k$ according to the following relationship (Trustorff et al. [3]):

$$
\text { Credit score }=\frac{1}{1+\exp \{-\vec{\beta} \cdot \vec{x}+\beta\}}
$$

Where and are predefined parameter values. Wosnitza and Leker [1] recently proposed a logistic regression model which allows the user to rank international corporates according to their credit qualities. They trained their logistic regression model on 618 financial reports of 312 international companies of which 156 had defaulted. Financial data from different industries except the financial industry was collected in order to obtain sufficient data. Their equation for calculating credit scores is based on the equity ratio $\left(\mathrm{ER}=\frac{\text { Equity }}{\text { Total assets }}\right)$ and the net debt ratio $\left(\mathrm{NDR}=\frac{\text { Financial liabilities }- \text { Cassh }}{\text { Capital employed }}\right):_{1}$

Credit score $=\frac{1}{1+\exp \{3.15 \cdot \mathrm{ER}-2.56 \cdot 10 \cdot \mathrm{NDR}-8.18 \cdot 10\}}$

${ }^{1}$ Here, the vector $\vec{x}_{k}$ is equal to $\left(\begin{array}{c}\mathrm{ER}_{k} \\ \mathrm{NDR}_{k}\end{array}\right)$.

\begin{tabular}{|l|l|l|l|}
\hline Company's name & Country of headquarter & Credit score & S\&P's rating \\
\hline Monsanto Co. & United States & 0.25 & $\mathrm{~A}+$ \\
\hline Sigma-Aldrich Corp. & United States & 0.22 & $\mathrm{~A}$ \\
\hline Solvay S.A. & Belgium & 0.31 & $\mathrm{~A}-$ \\
\hline AkzoNobel N.V. & Netherlands & 0.32 & BBB+ \\
\hline K+S AG & Germany & 0.33 & BBB \\
\hline Methanex Corp. & Canada & 0.35 & BBB- \\
\hline NewMarket Corp. & United States & 0.34 & BB+ \\
\hline Ashland Inc. & United States & 0.38 & BB \\
\hline EnergySolutions Inc. & United States & 0.41 & BB- \\
\hline Huntsman Corp. & United States & 0.53 & B+ \\
\hline OMNOVA Solutions Inc. & United States & 0.55 & B \\
\hline
\end{tabular}

Table 1: Comparison of two credit scores: The credit scores according to equation (1) and S\&P's credit ratings are listed for eleven chemical companies.
The higher the credit score, the lower is the credit quality of the respective company, and vice versa. Owing to the small value of its coefficient, the reader might get the idea to neglect the NDR. However, the incorporation of this financial ratio led to better classification results on test data than models without this ratio.

The purpose of the research note at hand is to compare the classification performances between the results of equation (2) and credit ratings from Standard and Poor's (S\&P's) for chemical companies. To this end, the ERs and the NDRs were computed for eleven chemical companies from their 2010 financial statements which were downloaded from Data-stream [4]. The credit quality of this sample was also evaluated by S\&P's. From each available rating class, one chemical company was randomly selected and included in the data set. The random character of the sample helps to avoid a selection bias, i.e., a systematic error in choosing the individuals to take part in the study. Within this sample, A+ denotes the best and B the worst credit quality. S\&P's credit ratings were obtained from S\&P's Global Credit Portal [5]. The credit score and S\&P's rating are summarized in Table 1 for each company.

In order to evaluate the classification performance of equation (1), we compare the rank order produced by the credit scores with the ranking according to S\&P's ratings. The two rank orders differ only for four companies. Monsanto Co. and Sigma-Aldrich Corp. as well as NewMarket Corp. and Methanex Corp. changed places, respectively. However, the distances between these two pairs are small.

The close match between the two rankings confirms equation (1). This equation seems to be able to rank large chemical companies according to their credit quality.

\section{References}

1. Wosnitza JH, Leker J (2014) Why credit risk markets are predestined for exhibiting log-periodic power law structures. Physica A: Statistical Mechanics and its Applications, 393: 427-449.

2. Credit ratings and complementary sources of credit quality information (2000) Basel Committee on Banking Supervision.

3. Trustorff, Jan-Henning, Paul Markus Konrad, Jens Leker (2011) Credit risk prediction using support vector machines. Review of Quantitative Finance and Accounting 36: 565-581.

4. Thomson Reuters (2014) Datastream.

5. Standard and Poor's (2011) Global Credit Portal.

*Corresponding author: Jan Henrik Wosnitza, Research Assistant, Institute of Business Administration, University of Muenster, Leonardo-Campus 1, 48149 Muenster, Germany Tel: +49 (251) 83 31813; E-mail: j.w@uni-muenster.de

Received February 18, 2014; Accepted April 25, 2014; Published April 28, 2014 Citation: Wosnitza JH (2014) Estimating the Credit Quality of Chemical Companies. J Bus Fin Aff 3:118 doi:10.4172/2167-0234.1000118

Copyright: @ 2014 Wosnitza JH. This is an open-access article distributed under the terms of the Creative Commons Attribution License, which permits unrestricted use, distribution, and reproduction in any medium, provided the original author and source are credited. 